\title{
microRNA-19a protects osteoblasts from dexamethasone via targeting TSC1
}

\author{
Gang Liu ${ }^{1, *}$, Feng-Li Chen ${ }^{2, *}$, Feng $\mathrm{Ji}^{1}$, Hao-Dong Fei ${ }^{1, *}$, Yue $\mathrm{Xie}^{1}$ and Shou-Guo \\ Wang $^{1}$ \\ ${ }^{1}$ Department of Orthopedics, Huai'an First People's Hospital, Nanjing Medical University, Huai'an, China \\ ${ }^{2}$ Clinical Laboratory, Huai'an First People's Hospital, Nanjing Medical University, Huai'an, China \\ * Co-first authors \\ Correspondence to: Feng Ji, email: huaiaifengjidr@163.com \\ Shou-Guo Wang, email: shouguoha126@126.com
}

Keywords: dexamethasone; osteoblasts; microRNA-19a; tuberous sclerosis complex 1 (TSC1); mTOR complex 1 (mTORC1)

Received: September 11,2017 Accepted: December 08, $2017 \quad$ Published: December 15, 2017

Copyright: Liv et al. This is an open-access article distributed under the terms of the Creative Commons Attribution License 3.0 (CC BY 3.0), which permits unrestricted use, distribution, and reproduction in any medium, provided the original author and source are credited.

\section{ABSTRACT}

Activation of mTOR complex 1 (mTORC1) could protect human osteoblasts from dexamethasone. Tuberous sclerosis complex 1 (TSC1) is mTORC1 upstream inhibitory protein. We demonstrate here that microRNA-19a ("miR-19a", -3p) targets the 3' untranslated regions of TSC1 mRNA. Expression of miR-19a downregulated TSC1 in OB-6 osteoblastic cells and primary human osteoblasts. miR-19a activated mTORC1 and protected human osteoblasts from dexamethasone. mTORC1 inhibition, by RAD001 or Raptor shRNA, almost completely abolished miR-19a-induced osteoblast cytoprotection against dexamethasone. Knockdown of TSC1 by targeted ShRNA similarly induced mTORC1 activation and protected osteoblasts. Moreover, miR-19a activated mTORC1-dependent NF-E2-related factor 2 ( Nrf2) signaling and inhibited dexamethasone-induced reactive oxygen species production in osteoblasts. Together, miR-19a protects human osteoblasts from dexamethasone possibly via targeting TSC1-mTORC1 signaling.

\section{INTRODUCTION}

Dexamethasone (Dex) shall induce direct injuries to human osteoblasts [1-3]. In the bones of Dex-taking patients, reduced number of osteoblasts and increased osteoblast cell apoptosis are often detected [4, 5]. Our group [6-12] and others [13-15] have been adding Dex to the cultured human osteoblasts in vitro, serving as a cellular model of glucocorticoid-induced osteoblast injuries.

Tuberous sclerosis complex 1 (TSC1)-TSC2 protein complex is an upstream inhibitor of mTOR complex 1 (mTORC1) $[16,17]$. Inhibition, silence, mutation or disruption of TSC1-TSC2 protein complex would induce mTORC1 activation, leading to phosphorylations of its downstream substrate proteins, including p70S6 kinase (p70S6K) and eukaryotic initiation factor 4E-binding protein 1 (4E-BP1) [18-20].

mTORC1 activation could promote cell survival $[16,17]$. Recent studies have implied that activation of mTORC1 could also protect osteoblasts from Dex $[6,21]$. The current study aims to induce mTORC1 activation by silencing TSC1 using microRNA strategy. miRNA can suppress expression of targeted-gene via binding to 3'-untranslated region (UTR) [22-24]. We show that microRNA-19a ("miR-19a") directly targets and downregulates TSC1, causing mTORC1 activation in human osteoblasts. Importantly, miR-19a expression protects human osteoblasts from Dex.

\section{RESULTS}

Forced-expression of miR-19a downregulates TSC1 in human osteoblasts

Results in Figure 1A showed that miR-19a ("-3p") targets the 3 '-untranslated region (UTR) of human TSC1 (at position 4878-4884). A lentiviral miR-19a expression vector ("LV-miR-19a") was established, which was 
introduced to OB-6 human osteoblastic cells. Cells were subjected to puromycin selection. Two lines of stable OB-6 cells with LV-miR-19a were established, which were named as "LV-miR-19a (Line-1)" and "LV-miR-19a (Line2)". The quantitative real-time PCR ("qRT-PCR") assay results in Figure 1B demonstrated that miR-19a ("-3p") expression level was dramatically increased in the two lines of OB-6 cells. (Figure 1B). Remarkably, TSC1 mRNA expression was decreased in the two lines of LV-miR-19a OB-6 cells (Figure 1C). Meanwhile, the TSC1 mRNA's 3' untranslated regions ( 3 '-UTR) luciferase activity was also inhibited after miR-19a expressing (Figure 1D), indicating that miR-19a directly targets TSC1 $m R N A$. $m R N A$ expression (Figure 1E) and 3'-UTR luciferase activity (Figure 1F) of TSC2, a protein that forms complex with TSC1, were unchanged by LV-miR-19a.

We also repeated the above experiments in the primary human osteoblasts. As demonstrated, forcedexpression of miR-19a by LV-miR-19a (Figure 1G) led to significant TSC1 $m R N A$ downregulation (Figure 1H). Meanwhile, TSC1 mRNA's 3'-UTR luciferase activity was also decreased by LV-miR-19a (Figure 1I). The non-sense scramble control microRNA ("miRC") didn't change miR19a nor TSC1/2 expressions in the osteoblasts (Figure 1B-1I). Collectively, these results indicate that forcedexpression of miR-19a induces TSC1 downregulation in human osteoblasts.

\section{miR-19a downregulates TSC1/2 and activates mTORC1 in human osteoblasts}

In the unstimulated condition, TSC1 forms a complex with TSC2, inhibiting mTORC1 [16, 17, 2527]. Inhibition of this complex, i.e. by AKT-dependent of phosphorylation of TSC2, will lead to downstream mTORC1 activation [16, 17, 25-27]. Importantly, TSC1 acts as a stabilizer of TSC 2 by protecting it from ubiquitindependent degradation [28]. TSC1 silence will lead to TSC2 ubiquitination and degradation [16, 17, 25-27]. The results in Figure 1 demonstrated that expression of miR-19a by LV-miR-19a caused TSC1 $m R N A$ depletion. Western blotting assay results in Figure 2A showed that TSC1 protein expression was also decreased in two lines of LV-miR-19a-expressing OB-6 cells. Importantly, TSC2 protein level was also reduced by LV-miR-19a (Figure 2B). Considering that TSC2 $m R N A$ was unchanged in LVmiR-19a OB-6 cells (Figure 1E), TSC2 downregulation by miR-19a should be due to the disruption of the TSC1-TSC2 complex. Remarkably, as shown in Figure 2B, phosphorylations of two key mTORC1 substrates, including 4EBP1 and p70S6K1 [19, 20, 29, 30], were significantly increased in miR-19a-expressing OB-6 cells, suggesting mTORC1 activation.

We repeated the experiments in the primary human osteoblasts. As demonstrated, introduction of LV-miR-19a also induced protein downregulation of TSC1 and TSC2 in the primary osteoblasts (Figure 2C). mTORC1 activation (p-4EBP1/p-S6K1) was significantly increase (Figure 2D). miRC, as expected, failed to change TSC $1 / 2$ expression nor mTORC1 activation (Figure 2A-2D).

To further confirm that TSC1 downregulation is the direct cause of mTORC1 activation in human osteoblasts, a TSC1-expression construct (see Method) was introduced to miR-19a-expressing OB-6 cells. The construct restored TSC1 expression (Figure 2E). Importantly, exogenous TSC1 expression in OB-6 cells almost completely blocked miR-19a-induced mTORC1 activation (tested by p-S6K1, Figure 2E). In miR-19a-expressing primary human osteoblasts, the TSC1 construct similarly restored TSC1 expression and abolished mTORC1 activation (Figure 2F). As expected, the TSC1 construct didn't affect miR19a expression by LV-miR-19a in the osteoblasts (Data not shown). These results imply that TSC1 silence should be the direct and primary cause of mTORC1 activation by miR-19a in human osteoblasts.

\section{miR-19a protects human osteoblasts from Dex}

Studies [6, 21] have shown that activation of mTORC1 could protect human osteoblasts from Dex, we therefore tested whether miR-19a expression could also exert similar functions. In line with our previous findings [6-11], treatment with Dex ( $1 \mu \mathrm{M}, 48$ hours $)$ in the control OB-6 cells induced potent viability reduction [Cell Counting Kit-8 (CCK-8) optic density (OD) decrease, Figure 3A), cell death [lactate dehydrogenase (LDH) release, Figure 3B] and apoptosis (Histone DNA ELISA OD increase, Figure 3C). Remarkably, Dex-induced cytotoxicity was dramatically attenuated in the two lines of OB-6 cells with LV-miR-19a (Figure 3A-3C). Thus, miR-19a expression indeed protected OB-6 cells from Dex. The similar results were also observed in the primary human osteoblasts, where LV-miR-19a attenuated Dexinduced cell viability reduction (Figure 3D) and cell death (Figure 3E). It should be noted that non-sense scramble control microRNA ("miRC") didn't inhibit Dex-induced cytotoxicity in human osteoblasts.

\section{mTORC1 activation is required for miR-19a- mediated osteoblast cytoprotection against Dex}

In order to prove that mTORC1 activation is required for miR-19a-mediated osteoblast cytoprotection, pharmacological and genetic methods were employed to block mTORC1 activation. RAD001, a well-known specific mTORC1 inhibitor, was applied [31, 32]. Since long-term RAD001 treatment might also inhibit mTOR complex 2 (mTORC2) activation [33], shRNA method was utilized to knockdown Raptor in OB-6 cells. The latter (Raptor) is key component of mTORC1 [34-36]. Notably, RAD001 or Raptor shRNA almost completely blocked mTORC1 activation (p-S6K1/p-4EBP1) in OB-6 
cells with LV-miR-19a ("Line1", Figure 4A). The applied Raptor shRNA potently silenced Raptor in OB-6 cells (Figure 4A). Dex-induced cytotoxicity in OB-6 cells was intensified by RAD001 and Raptor shRNA, leading to increased viability reduction (Figure 4B) and apoptosis (Figure 4C). Thus, mTORC1 activation was cytoprotective in Dex-treated OB-6 cells. Remarkably, miR-19a-induced osteoblast cytoprotection against Dex was almost completely nullified by RAD001 or Raptor shRNA (Figure $4 B$ and $4 C$ ). Similarly in the primary human osteoblasts, mTORC1 blockage by RAD001 (Figure 4D) intensified
Dex-induced injuries and almost reversed miR-19ainduced anti-Dex actions (Figure 4E and 4F). These results suggest that mTORC1 activation is required for miR-19ainduced osteoblast cytoprotection against Dex.

\section{TSC1 is the primary target of miR-19a in mediating its cytoprotective activity in osteoblasts}

If TSC1 is the primary target protein of miR-19a, TSC1 knockdown shall also protect osteoblasts from

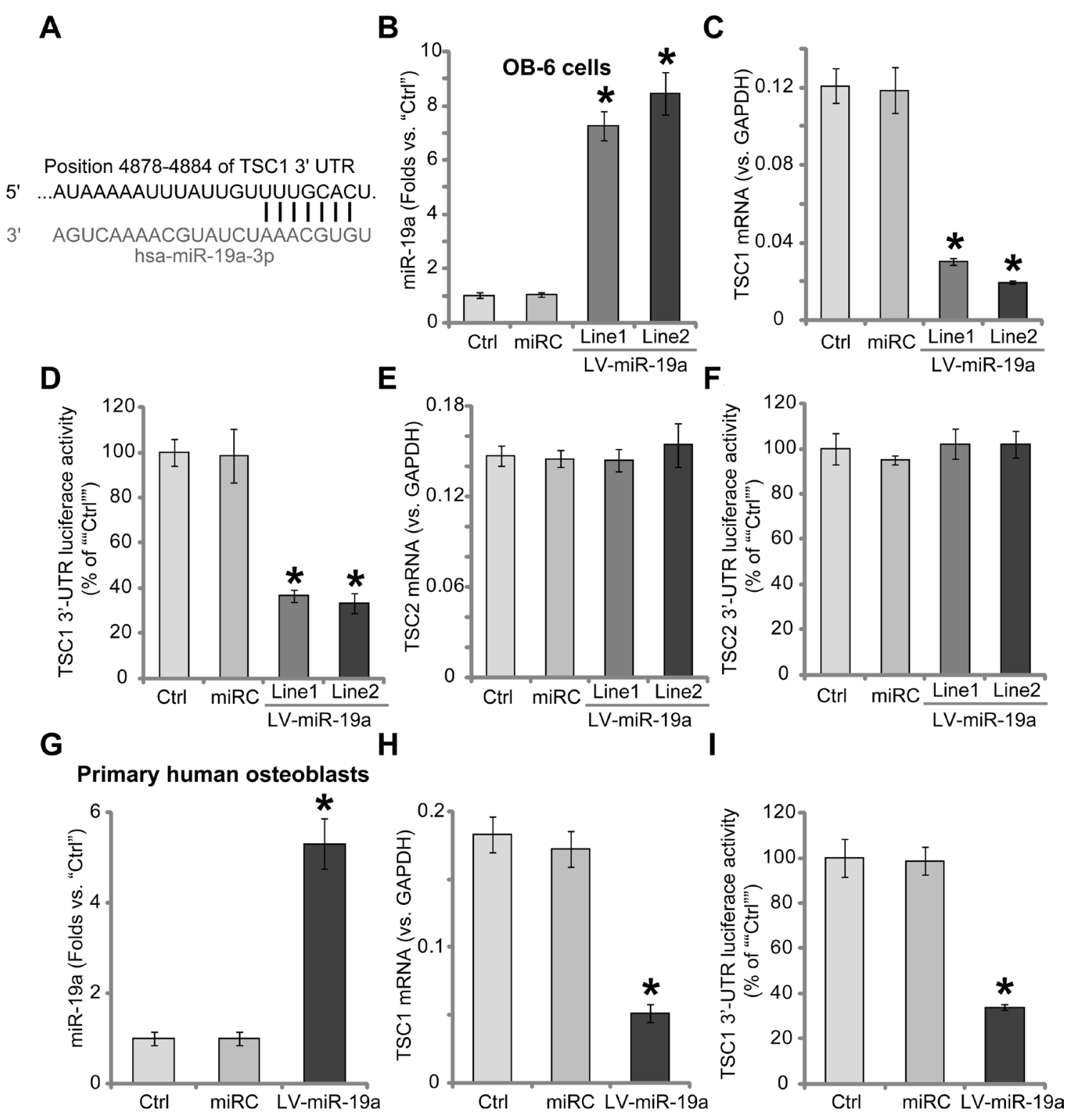

Figure 1: Forced-expression of miR-19a downregulates TSC1 in human osteoblasts. microRNA-19a-3p ("miR-19a") putatively targets the 3'-UTR of human TSC1 mRNA (A). Stable OB-6 osteoblastic cells, expressing the lentiviral miR-19a expression vector ("LV-miR-19a", two lines, "Line1/2"), non-sense scramble control microRNA ("miRC"), or the parental control OB-6 cells ("Ctrl"), were subjected to quantitative real-time PCR ("qRT-PCR") assay of miR-19a ("-3p") and TSC1/2 mRNA expressions (B, C and E); TSC1/2 $m R N A$ 3'-UTR luciferase activity assay was also tested (D and F). Primary human osteoblasts were infected with the LV-miR-19a or miRC for 48 hours, miR-19a (“-3p") expression (G), TSC1 mRNA level (H) and TSC1 mRNA 3'-UTR luciferase activity (I) were tested similarly. Data were expressed as mean $\pm \mathrm{SD}(n=5)$. ${ }^{*}<0.05 v s$. "Ctrl". Experiments in this figure were repeated five times, and similar results were obtained. 
Dex. To test this hypothesis, shRNA method was again utilized to knockdown TSC1. As demonstrated, the applied lentiviral TSC1 shRNA (purchased from Santa Cruz Biotech) potently downregulated TSC1 in OB-6 cells (Figure 5A). TSC2 protein level was also downregulated (Figure 5A). Correspondingly, mTORC1 activation, or
p-S6K1, was boosted (Figure 5A). In the TSC1-shRNAexpressing OB-6 cells, introduction of LV-miR-19a failed to further change TSC1 expression and mTOR activation (Figure 5A). Importantly, OB-6 cells with TSC1 shRNA were also protected from Dex, showing reduced viability reduction (Figure 5B) and cell death (Figure 5C).

C

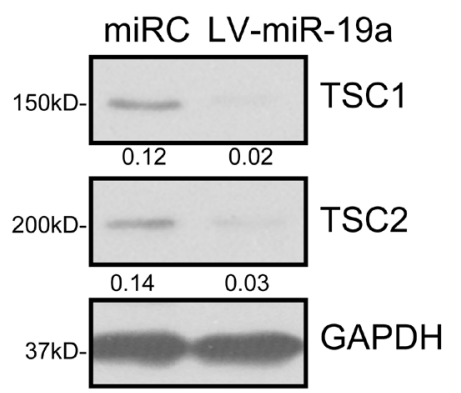

D

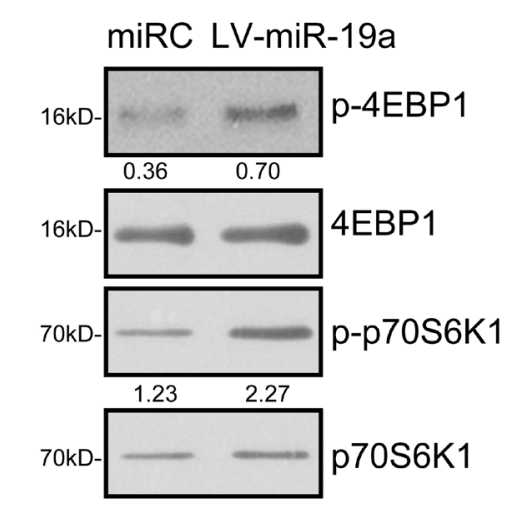

$\mathbf{E}$

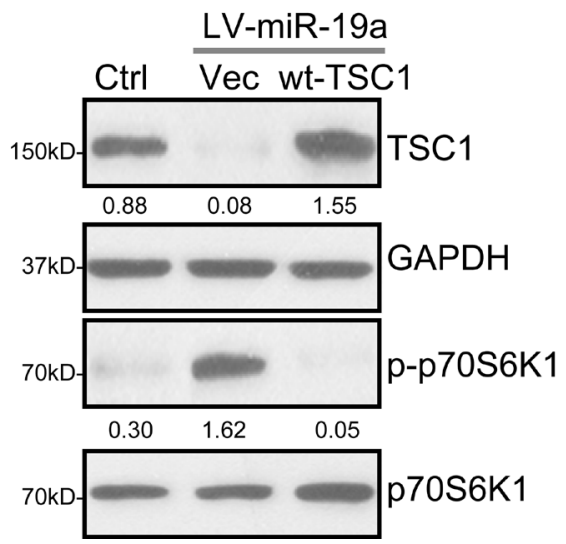

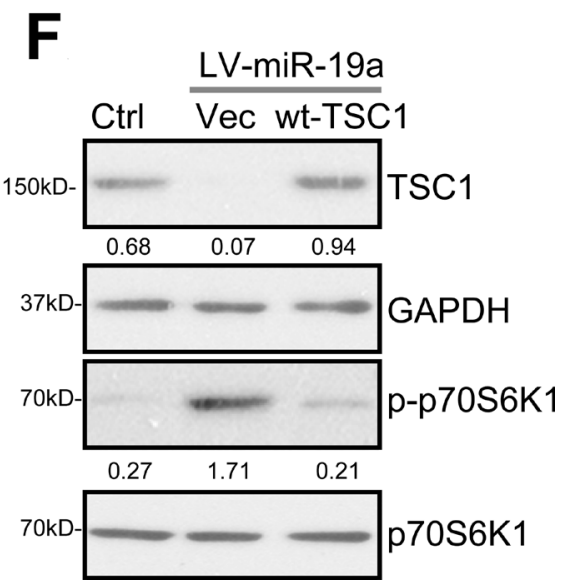

Figure 2: miR-19a downregulates TSC1/2 and activates mTORC1 in human osteoblasts. Stable OB-6 cells, expressing the lentiviral miR-19a expression vector ("LV-miR-19a", two lines, "Line1/2"), non-sense scramble control microRNA ("miRC"), or the parental control OB-6 cells ("Ctrl"), were subjected to Western blotting assay of listed proteins (A and B). Primary human osteoblasts were infected with LV-miR-19a or miRC for 48 hours, expressions of listed proteins were shown (C and D). Stable OB-6 cells with LV-miR19a ("Line1") (E), as well as the primary human osteoblasts with LV-miR-19a (F), were further transfected with TSC1 cDNA construct ("wt-TSC1", $0.20 \mu \mathrm{g} / \mathrm{mL}$, for 48 hours) or the empty vector (pSuper-puro-EGFP, "Vec"), total cell lysates were subjected to Western blotting assay of listed proteins. The indicated protein band was quantified (total gray), and its value was normalized to the loading control. Experiments in this figure were repeated four times, and similar results were obtained. 
Intriguingly, expression of miR-19a (by LV-miR-19a) in the TSC1 shRNA-expressing OB-6 cells was unable to further protect cells from Dex (Figure 5B and 5C). These results together indicate that TSC1 is the primary target of miR-19a in mediating its osteoblast cytoprotection.

\section{miR-19a activates Nrf2 signaling in human osteoblasts}

It has been previously shown that Dex induces reactive oxygen species (ROS) production, which is required for subsequent osteoblast cell death and apoptosis [7, 11, 13, 37]. Activation of mTORC1 could exert anti-oxidant activity via activating downstream NFE2-related factor $2(\mathrm{Nrf} 2)[38,39]$. We thus tested the potential effect of miR-19a on Nrf2 signaling activation. The qRT-PCR assay results in Figure 6A confirmed that expression of miR-19a by LV-miR-19a significantly increased $m R N A$ expressions of Nrf2-regulated genes, including heme oxygenase 1 (HO-1), NAD $(\mathrm{P}) \mathrm{H}$ quinone oxidoreductase 1 (NQO-1) and $\gamma$-glutamylcysteine synthetase catalytic subunit $(G C L-C)$. HO-1, NQO-1 and GCL-C protein expressions were also upregulated in LVmiR-19a OB-6 cells (Figure 6B). Nrf2 $m R N A$ level was unchanged by LV-miR-19a (Figure 6A), yet its protein level was significantly increased (Figure 6B). These results suggest that miR-19a expression induced Nrf2 protein stabilization, which is necessary for its activation $[40,41]$. Notably, inhibition of mTORC1, by RAD001 or Raptor shRNA, almost completely blocked LV-miR19a-induced $m R N A$ expression of Nrf2-regulated genes, including HO-1 (Figure 6C) and NQO-1 (Figure 6D), indicating that $\mathrm{mTORC} 1$ activation is required for miR$19 \mathrm{a}-$ mediated Nrf2 activation in osteoblasts. Importantly, results showed that Dex-induced ROS production was largely attenuated following miR-19a expression in OB-6 cells (Figure 6E). Thus, miR-19a expression apparently activated mTORC1-dependent Nrf2 signaling and inhibited Dex-induced oxidative stresses in OB-6 cells. In the primary human osteoblasts, introduction of LV-miR19a also induced $m R N A$ expressions of $H O-1, N Q O-1$ and $G C L-C$ (Figure 6F).

\section{DISCUSSION}

In the current study, we propose that miR-19a is a TSC1-targeting miRNA in human osteoblasts. Expression of miR-19a downregulated TSC1 and activated mTORC1 signaling in both OB-6 osteoblastic cells and primary human osteoblasts. Significantly, miR-19a protected

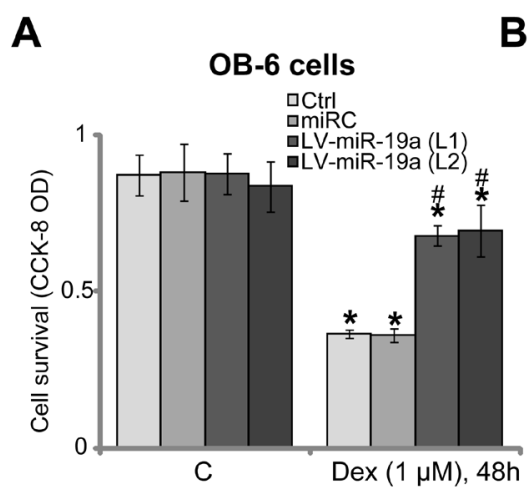

D
B
C

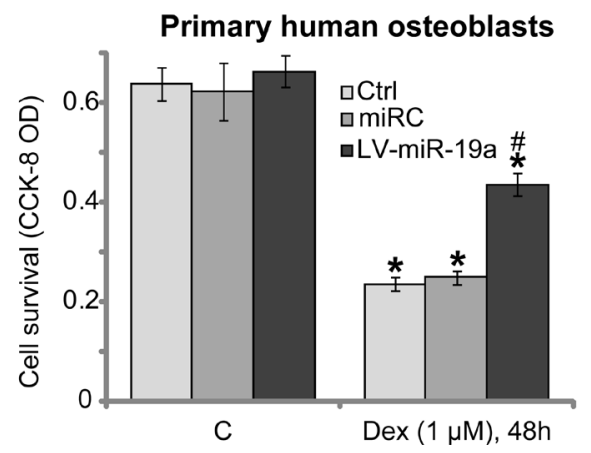

E

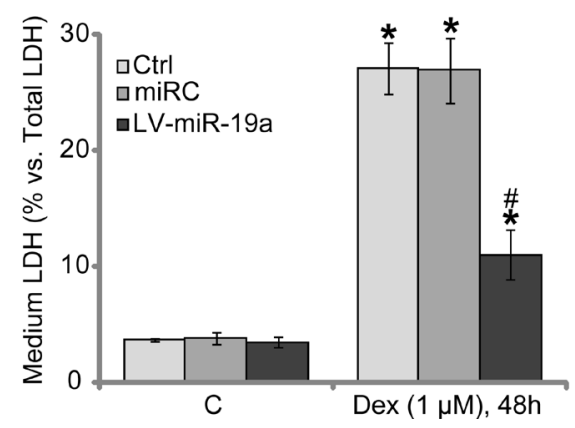

Figure 3: miR-19a protects human osteoblasts from Dex. OB-6 osteoblastic cells (A-C) or the primary human osteoblasts (D-E), with miR-19a expression vector ("LV-miR-19a") or the non-sense scramble control microRNA ("miRC"), as well as the parental control cells ("Ctrl"), were treated with/out Dex $(1 \mu \mathrm{M})$ for 48 hours, cell viability (CCK-8 assay, A and D), cell death (LDH release assay, B and E) and apoptosis (Histone DNA ELISA assay, C) were examined. Data were expressed as mean \pm SD $(n=5)$. "C" stands for untreated control group. ${ }^{*} p<0.05$ vs. "C". " $p<0.05$ vs. Dex treatment of "miRC" cells. Experiments in this figure were repeated three times, and similar results were obtained. 
human osteoblasts from Dex, and activation of mTORC1 is required for the process. $\mathrm{mTORC} 1$ inhibition, by RAD001 or Raptor shRNA, almost completely abolished miR19a-induced osteoblast cytoprotection. Similarly, TSC1
shRNA activated mTORC1 and protected osteoblasts from Dex. Notably, miR-19a was in valid in TSC1-silenced OB-6 cells. These results imply that TSC1 should be the primary and direct target protein of miR-19a in mediating
A

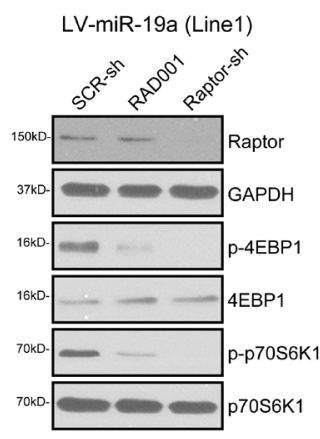

D

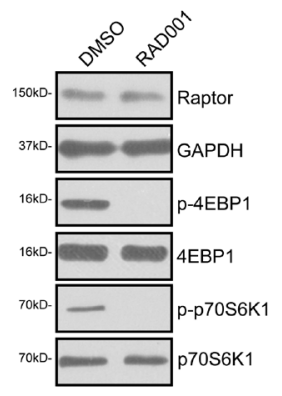

B

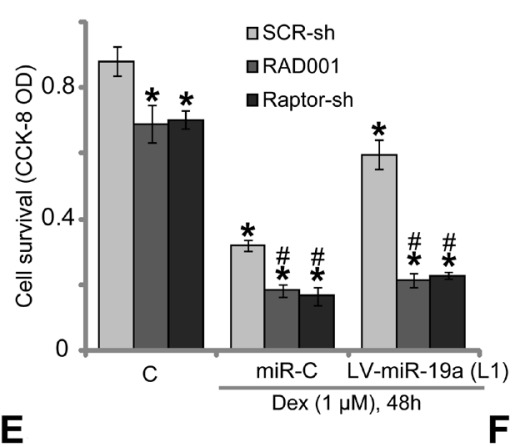

$\mathbf{E}$

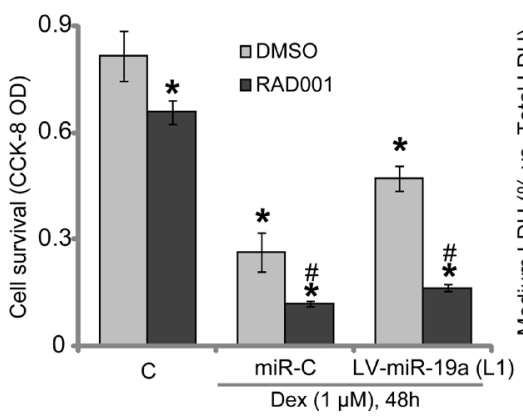

C

$\mathbf{F}$
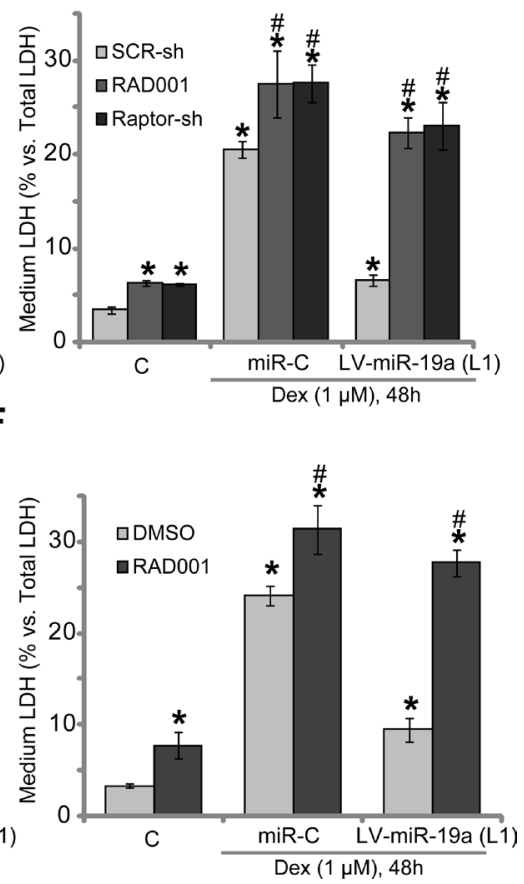

Figure 4: mTORC1 activation is required for miR-19a-mediated osteoblast cytoprotection against Dex. OB-6 cells, expressing the lentiviral miR-19a expression vector ("LV-miR-19a", Line1) or the non-sense scramble control microRNA ("miRC"), were further infected with lentiviral scramble control shRNA ("SCR-sh") or Raptor shRNA ("Raptor-sh") for 24 hours, cells were treated with/ out Dex $(1 \mu \mathrm{M})$, or plus RAD001 (200 nM); Expressions of listed proteins were shown (A, 1 hour after Dex treatment); Cell survival (B) and death $(\mathbf{C})$ were tested 48 hours after Dex treatment. Primary human osteoblasts were infected with the LV-miR-19a or miRC for 48 hours, cells were treated with/out Dex $(1 \mu \mathrm{M})$, or plus RAD001 (200 nM); Expressions of listed proteins were shown $(\mathbf{D}, 1$ hour after Dex treatment); Cell survival $(\mathbf{E})$ and death $(\mathbf{F})$ were tested 48 hours after Dex treatment. Data were expressed as mean $\pm \mathrm{SD}(n=5)$. "C" stands for untreated control group. " $p<0.05$ vs. "C". $p<0.05$ vs. Dex treatment of "SCR-sh" cells (B and C) or "DMSO" cells (E and F). Experiments in this figure were repeated three times, and similar results were obtained.
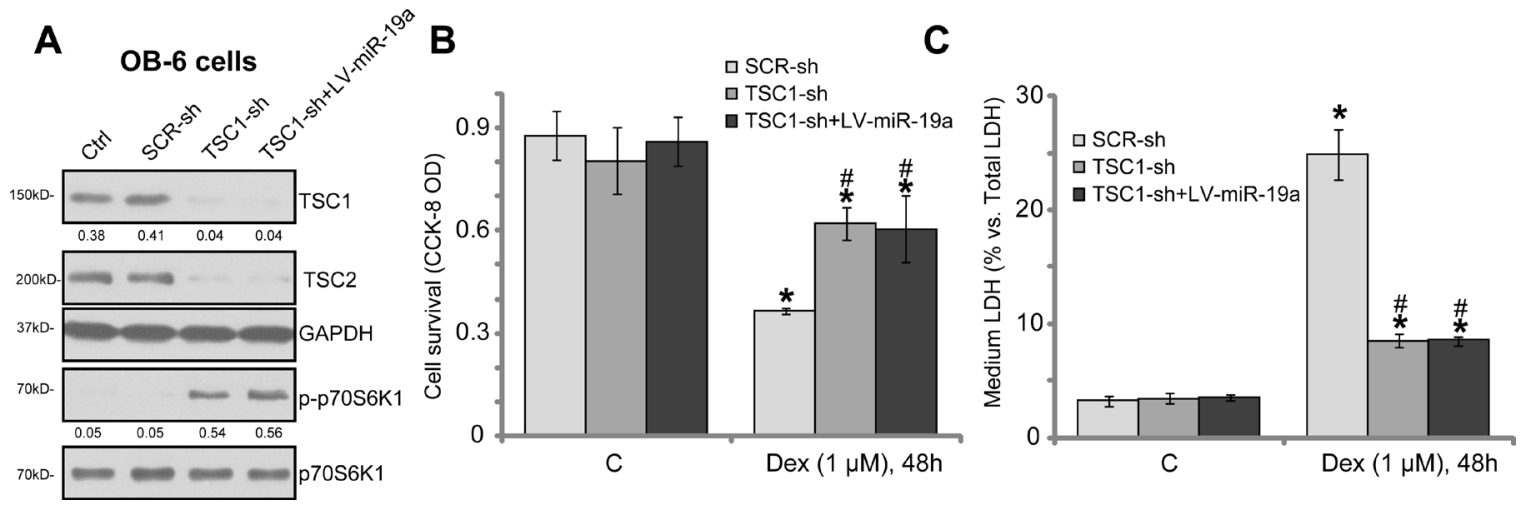

Figure 5: TSC1 is the primary target of miR-19a in mediating its cytoprotective activity in osteoblasts. The OB-6 osteoblastic cells were infected with lentiviral scramble control shRNA ("SCR-sh"), TSC1 shRNA ("TSC1-sh") or plus lentiviral miR-19a expression vector ("+LV-miR-19a"), cells were further selected by puromycin, expressions of listed proteins were shown (A). The abovementioned cells were also treated with/out Dex $(1 \mu \mathrm{M})$ for 48 hours, cell survival $(\mathbf{B})$ and death $(\mathbf{C})$ were tested. The indicated protein band wan quantified, and its value was normalized to the loading control (A). Data were expressed as mean \pm SD ( $n=5)$. "Ctrl" stands for parental control cells. "C" stands for untreated control group. " $p<0.05 v s$. "C". $p<0.05$ vs. Dex treatment of "SCR-sh" cells. Experiments in this figure were repeated three times, and similar results were obtained. 
its cytoprotective activity in osteoblasts. Indeed, we show that TSC1 mRNA's 3'-UTR luciferase assay was also largely attenuated after miR-19a expression.

Our group [7-9, 11] and others have demonstrated that Dex induces reactive oxygen species (ROS) production, which mediates following osteoblast cell apoptosis [9, 42]. Reversely, inhibition of oxidative stress protects osteoblasts from Dex [42-44]. A novel AMPactivated protein kinase (AMPK) activator compound 13 protected osteoblasts from Dex via inhibiting ROS production [9]. Similarly, activation of EGFR-Akt-Nrf2 signaling by icariside II inhibited Dex-induced oxidative stress and osteoblast cell apoptosis [11]. Additionally, SC79-induced osteoblast cytoprotection against Dex
A
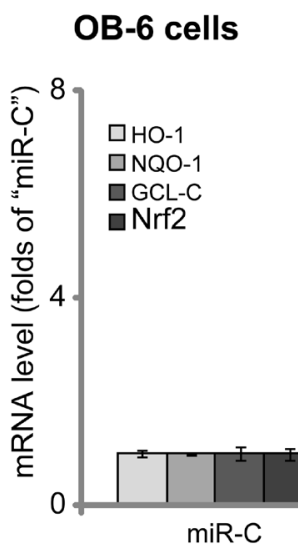

C

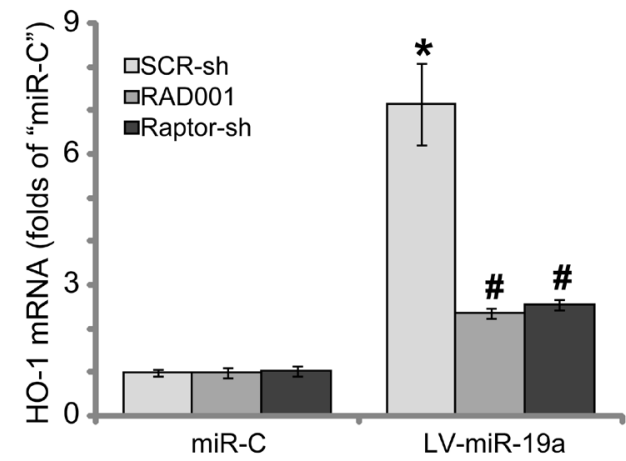

E

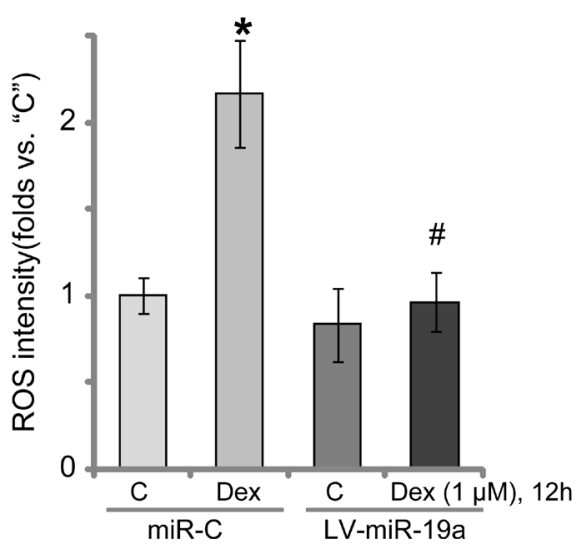

B

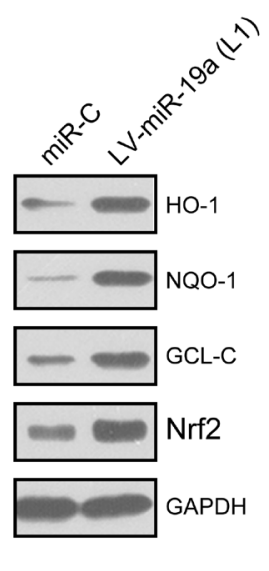

D

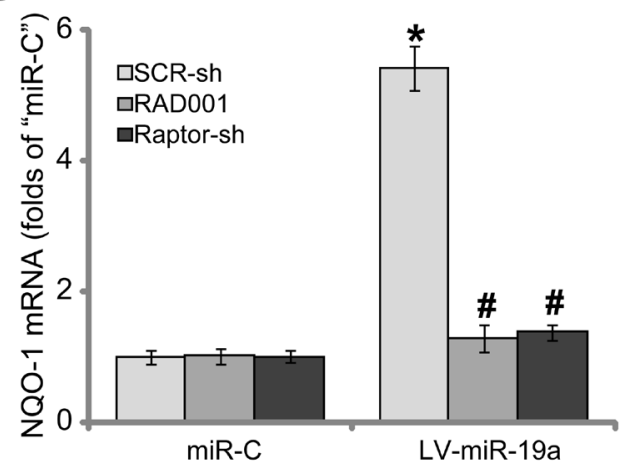

F

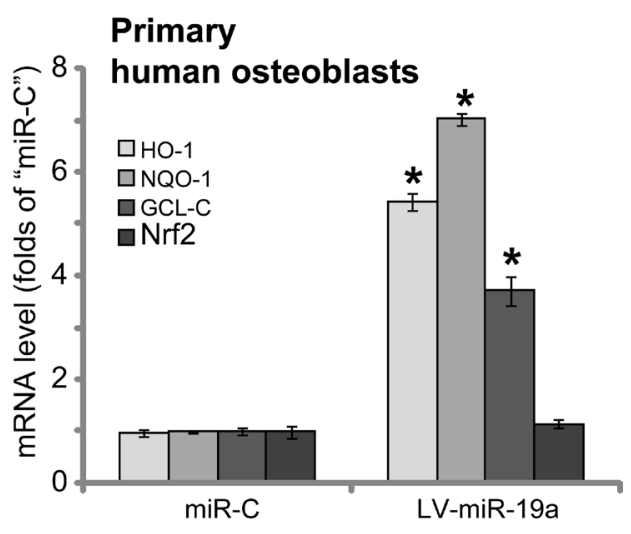

Figure 6: miR-19a activates Nrf2 signaling in human osteoblasts. OB-6 osteoblastic cells (A and B) or primary human osteoblasts (F), with the lentiviral miR-19a expression vector ("LV-miR-19a") or non-sense scramble control microRNA ("miRC"), were subjected to qRT-PCR assay (A and F) and Western blotting assay (B) of listed Nrf2 pathway genes; The OB-6 cells were also treated with/out Dex $(1 \mu \mathrm{M})$ for 12 hours, relative ROS intensity was analyzed by the DCFH-DA fluorescent dye assay (E). OB-6 cells, with "LV-miR-19a" or "miRC", were further infected with lentiviral scramble control shRNA ("SCR-sh") or Raptor shRNA ("Raptor-sh") for 24 hours, or plus RAD001 (200 nM) co-treatment, qRT-PCR assay was applied to test HO-1 mRNA (C) and NQO-1 mRNA (D); Data were expressed as mean $\pm \mathrm{SD}(n=5)$. "C" stands for untreated control group. ${ }^{*} p<0.05$ vs. "miRC". $p<0.05$ vs. "SCR-sh" cells $(\mathbf{C}$ and $\mathbf{D}) . " p<0.05 v s$. Dex treatment of "miRC" cells (E). Experiments in this figure were repeated three times, and similar results were obtained. 
was also due to ROS scavenging [37]. In the current study, miR-19a expression inhibited Dex-induced ROS production in osteoblasts. This could explain the superior cytoprotective activity of miR-19a against Dex in human osteoblasts.

The transcription factor Nrf2 signaling is one key cellular defense mechanism against oxidative stress [40, 45]. Recent studies have proposed that mTORC1 could be a upstream signaling for $\mathrm{Nrf2}$ activation [38, 39]. Activated mTORC1 could induce Nrf2 phosphorylation at Ser-40, causing its departure from Keap1. This will lead to Nrf2 stabilization, its nuclear translocation and activation [38, 39]. Here, expression of miR-19a induced Nrf2 stabilization and expression of Nrf2-regulated genes (HO-1, NQO-1 and $G C L-C$ ). Inhibition of mTORC1, by RAD001 or Raptor shRNA, almost completely blocked miR-19a-induced mRNA expression of Nrf2regulated genes. These results indicate that miR-19ainduced mTORC1 activation could possibly activate Nrf2 signaling, which likely inhibits Dex-induced oxidative stress. The detailed mechanism may warrant further characterizations.

\section{CONCLUSIONS}

Taken together, miR-19a protects human osteoblasts from Dex possibly via targeting TSC1-mTORC1 signaling.

\section{METHODS}

\section{Chemicals and reagents}

Dexamethasone (Dex), puromycin and RAD001 were provided by Sigma Aldrich (Nantong, China). Cell culture reagents were purchased from Gibco Co. (Nantong, China). All the antibodies utilized in this study were obtained from Cell Signaling Technology (Nanjing, China). Lipofectamine 2000 reagent was provided by Invitrogen (Suzhou, China). All the primers were from Genepharm (Shanghai, China) unless otherwise mentioned.

\section{OB-6 cell culture}

The adherent OB-6 osteoblastic cells were seeded at a density of 5000 cells $/ \mathrm{cm}^{2}$ and were cultured in $\alpha$-minimal essential medium (MEM) with 10\% fetal bovine serum (FBS), plus $1 \%$ each of penicillin, streptomycin, and glutamine.

\section{Culture of primary human osteoblasts}

The redundant trabecular bone fragments were obtained from the written-informed consent healthy donors at Huai'an First People's Hospital (Huai'an, Jiangsu,
China). The trabecular bone fragments were minced and washed 2-3 times with warm PBS, which were then digested using DNase and collagenase type II. After $2 \mathrm{~h}$ digestion at $37^{\circ} \mathrm{C}$, bone debris was separated by using a $70-\mu \mathrm{m}$ nylon mesh (Falcon). Cells were precipitated by centrifugation, washed several times to remove excess collagenase and DNase enzymes. The primary osteoblasts were then resuspended in $\alpha$-MEM supplemented with $10 \% \mathrm{FBS}$, which were plated at $2 \times 10^{4}$ cells $/ \mathrm{cm}^{2}$. After confluence, cells were trypsinized, resuspended, and seeded at $1.5 \times 10^{4} / \mathrm{cm}^{2}$. The primary osteoblasts at passage 3-5 were utilized for further experiments. The protocols were approved by Ethics Review Board of Nanjing Medical University.

\section{RNA isolation and qRT-PCR}

The protocols of quantitative real-time PCR ("qRTPCR") using the SYBR green kit and ABI-7600 FAST system (Applied Biosystems, Shanghai, China) were described previously $[7,10,11]$. The $m R N A$ primers for human TSC1 were described [46]. The $m R N A$ primers for the human tuberin (TSC2) (catalog no. Hs00241068_s1) were purchased from Applied Biosystems (Shanghai, China). The $m R N A$ primers of Nrf2-regulated genes, including $\mathrm{Nrf2}, \mathrm{HO}-1$, GCL-C and $\mathrm{NQO}-1$, as well as $m R N A$ primers of GAPDH were described [38, 39, 47, 48]. For analyzing miR, the miR was converted to cDNA using the First-Strand Synthesis Kit (SABiosciences, Frederick, MD). Human miR-19a (“-3p") analysis was tested via the qRT-PCR assay using stem-loop primers, designed as previously described [49].

\section{Forced-expression of miR-19a}

The lentiviral pSuper-GFP-puro-miR-19a expression vector ("LV-miR-19a") was designed (based on the descried sequence [49]) and synthesized by Genepharm (Shanghai, China). The human osteoblasts were infected with LV-miR-19a lentivirus or the scramble non-sense control miRNA ("miRC"). Stable cells were selected by puromycin $(0.5 \mu \mathrm{g} / \mathrm{mL}$, Sigma $)$. Expression of miR-19a ("-3p") in the resulting cells was tested by qRTPCR assay.

\section{TSC1 3'-UTR luciferase assay}

TSC1 mRNA's 3'-UTR was amplified via the primers as described [50], which was sub-cloned into pMIR-Report plasmid [50]. The reporter plasmid was then utilized as template to generate a miR-19a response element. The plasmid with the perfect match contain the complementary sequences of the mature miR-19a$3 p$ behind the firefly luciferase gene [49]. OB-6 cells were transfected with luciferase reporter plasmid by Lipofectamine 2000. The luciferase activity was measured 
24 hours after transfection using the Dual-Luciferase reporter assay system (Promega, Shanghai, China).

Cell survival, death and apoptosis assays. As described [7-11], cell survival, death and apoptosis were tested by the Cell Counting Kit-8 (CCK-8, Dojindo Laboratories, Kumamoto, Japan) assay, lactate dehydrogenase (LDH) release assay (Biyuntian, Wuxi, China) and the histone-DNA ELISA cell apoptosis plus kit (Roche, Shanghai, China), respectively [7-11].

\section{Western blotting assay}

The detailed protocols for Western blotting assay were described in detail in our previous studies $[6,7,11,12]$. The total gray of the protein band was quantified via ImageJ software, and the value was normalized to the loading control.

\section{ShRNA}

The lentiviral TSC1 shRNA (catalog no. sc37437-V), the lentiviral Raptor shRNA (catalog no. sc44069-V), and the lentiviral scramble control shRNA (catalog no.sc-108060) were all purchased from the Santa Cruz Biotech (Shanghai, China). The lentiviral shRNA (10 $\mu \mathrm{L}$ virus/ $1 \mathrm{~mL}$ medium, per well) was added directly to the cultured osteoblasts for 24 hours. Afterwards, puromycin $(0.5 \mu \mathrm{g} / \mathrm{mL}$, Sigma $)$ was added to select stable cells. Knockdown of the targeted-protein in the stable cells was verified via Western blotting assay.

\section{Exogenous TSC1 expression}

The full length human TSC1 $c D N A$ was synthesized, sequence-verified and provided by Genepharm (Shanghai, China), which was inserted into the pSuper-puro-EGFP vector (Addgene, Shanghai, China). The construct was transfected to miR-19a-expressing osteoblasts via Lipofectamine 2000 transfection. Expression of TSC1 in the resulting cells was verified by the Western blotting assay.

\section{Reactive oxygen species (ROS) assay}

As previously described [7-9, 11, 12], cellular ROS content was tested by the dichloro-dihydro-fluorescein diacetate (DCFH-DA) fluorescent dye (Invitrogen, Shanghai, China) assay.

\section{Statistical analysis}

All values were expressed as means \pm standard deviation (SD). The statistical significance of differences among groups were determined by one-way analysis of variance (ANOVA) followed by the Tukey's post hoc multiple comparison tests. $p<0.05$ was considered significant.

\section{Author contributions}

All authors carried out the experiments, participated in the design of the study and performed the statistical analysis, participated in its design and coordination and helped to draft the manuscript.

\section{ACKNOWLEDGMENTS}

This work is supported by the National Natural Science Foundation (81672170).

\section{CONFLICTS OF INTEREST}

The listed authors have no conflicts of interest.

\section{REFERENCES}

1. den Uyl D, Bultink IE, Lems WF. Advances in glucocorticoidinduced osteoporosis. Curr Rheumatol Rep. 2011; 13:233-40. https://doi.org/10.1007/s11926-011-0173-y.

2. Weinstein RS. Clinical practice. Glucocorticoid-induced bone disease. N Engl J Med. 2011; 365:62-70. https://doi. org/10.1056/NEJMcp1012926.

3. Kerachian MA, Seguin C, Harvey EJ. Glucocorticoids in osteonecrosis of the femoral head: a new understanding of the mechanisms of action. J Steroid Biochem Mol Biol. 2009; 114:121-8. https://doi.org/10.1016/j.jsbmb.2009.02.007.

4. Ding H, Wang T, Xu D, Cha B, Liu J, Li Y. Dexamethasoneinduced apoptosis of osteocytic and osteoblastic cells is mediated by TAK1 activation. Biochem Biophys Res Commun. 2015; 460:157-63. https://doi.org/10.1016/j. bbrc.2015.02.161.

5. Yun SI, Yoon HY, Jeong SY, Chung YS. Glucocorticoid induces apoptosis of osteoblast cells through the activation of glycogen synthase kinase 3beta. J Bone Miner Metab. 2009; 27:140-8. https://doi.org/10.1007/ s00774-008-0019-5.

6. Zhao S, Chen C, Wang S, Ji F, Xie Y. MHY1485 activates mTOR and protects osteoblasts from dexamethasone. Biochem Biophys Res Commun. 2016; 481:212-8. https:// doi.org/10.1016/j.bbrc.2016.10.104.

7. Ji F, Mao L, Liu Y, Cao X, Xie Y, Wang S, Fei H. K6PC-5, a novel sphingosine kinase 1 (SphK1) activator, alleviates dexamethasone-induced damages to osteoblasts through activating SphK1-Akt signaling. Biochem Biophys Res Commun. 2015; 458:568-75. https://doi.org/10.1016/j. bbrc.2015.02.007.

8. Guo S, Xie Y, Fan JB, Ji F, Wang S, Fei H. alpha-Melanocyte stimulating hormone attenuates dexamethasone-induced osteoblast damages through activating melanocortin receptor 4-SphK1 signaling. Biochem Biophys Res Commun. 2016; 469:281-7. https://doi.org/10.1016/j.bbrc.2015.11.104.

9. Guo S, Mao L, Ji F, Wang S, Xie Y, Fei H, Wang XD. Activating AMP-activated protein kinase by an alphal 
selective activator compound 13 attenuates dexamethasoneinduced osteoblast cell death. Biochem Biophys Res Commun. 2016; 471:545-52. https://doi.org/10.1016/j. bbrc.2016.02.036.

10. Guo S, Chen C, Ji F, Mao L, Xie Y. PP2A catalytic subunit silence by microRNA-429 activates AMPK and protects osteoblastic cells from dexamethasone. Biochem Biophys Res Commun. 2017; 487:660-5. https://doi.org/10.1016/j. bbrc.2017.04.111.

11. Liu W, Mao L, Ji F, Chen F, Wang S, Xie Y. Icariside II activates EGFR-Akt-Nrf2 signaling and protects osteoblasts from dexamethasone. Oncotarget. 2017; 8:2594-603. https://doi.org/10.18632/oncotarget.13732.

12. Liu W, Mao L, Ji F, Chen F, Hao Y, Liu G. Targeted activation of AMPK by GSK621 ameliorates H2O2-induced damages in osteoblasts. Oncotarget. 2017; 8:10543-52. https://doi.org/10.18632/oncotarget.14454.

13. Zhen YF, Wang GD, Zhu LQ, Tan SP, Zhang FY, Zhou XZ, Wang XD. P53 dependent mitochondrial permeability transition pore opening is required for dexamethasoneinduced death of osteoblasts. J Cell Physiol. 2014; 229:1475-83. https://doi.org/10.1002/jcp.24589.

14. Fan JB, Liu W, Yuan K, Zhu XH, Xu DW, Chen JJ, Cui ZM. EGFR trans-activation mediates pleiotrophin-induced activation of Akt and Erk in cultured osteoblasts. Biochem Biophys Res Commun. 2014; 447:425-30. https://doi. org/10.1016/j.bbrc.2014.04.002.

15. Li H, Qian W, Weng X, Wu Z, Zhuang Q, Feng B, Bian Y. Glucocorticoid receptor and sequential P53 activation by dexamethasone mediates apoptosis and cell cycle arrest of osteoblastic MC3T3-E1 cells. PLoS One. 2012; 7:e37030. https://doi.org/10.1371/journal.pone.0037030.

16. Inoki $\mathrm{K}$, $\mathrm{Li} \mathrm{Y}$, Zhu $\mathrm{T}$, Wu J, Guan KL. TSC2 is phosphorylated and inhibited by Akt and suppresses mTOR signalling. Nat Cell Biol. 2002; 4:648-57. https://doi. org/10.1038/ncb839.

17. Mak BC, Yeung RS. The tuberous sclerosis complex genes in tumor development. Cancer Invest. 2004; 22:588-603.

18. Sabatini DM. mTOR and cancer: insights into a complex relationship. Nat Rev Cancer. 2006; 6:729-34. https://doi. org/10.1038/nrc1974.

19. Laplante M, Sabatini DM. mTOR signaling in growth control and disease. Cell. 2012; 149:274-93. https://doi. org/10.1016/j.cell.2012.03.017.

20. Saxton RA, Sabatini DM. mTOR Signaling in Growth, Metabolism, and Disease. Cell. 2017; 168:960-76. https:// doi.org/10.1016/j.cell.2017.02.004.

21. Fan JB, Liu W, Zhu XH, Yuan K, Xu DW, Chen JJ, Cui ZM. EGFR-AKT-mTOR activation mediates epiregulininduced pleiotropic functions in cultured osteoblasts. Mol Cell Biochem. 2015; 398:105-13. https://doi.org/10.1007/ s11010-014-2210-4.

22. Thomson DW, Dinger ME. Endogenous microRNA sponges: evidence and controversy. Nat Rev Genet. 2016; 17:272-83. https://doi.org/10.1038/nrg.2016.20.
23. Jonas S, Izaurralde E. Towards a molecular understanding of microRNA-mediated gene silencing. Nat Rev Genet. 2015; 16:421-33. https://doi.org/10.1038/nrg3965.

24. Pasquinelli AE. MicroRNAs and their targets: recognition, regulation and an emerging reciprocal relationship. Nat Rev Genet. 2012; 13:271-82. https://doi.org/10.1038/nrg3162.

25. Inoki K, Zhu T, Guan KL. TSC2 mediates cellular energy response to control cell growth and survival. Cell. 2003; 115:577-90. https://doi.org/S0092867403009292.

26. Ma L, Chen Z, Erdjument-Bromage H, Tempst P, Pandolfi PP. Phosphorylation and functional inactivation of TSC2 by Erk implications for tuberous sclerosis and cancer pathogenesis. Cell. 2005; 121:179-93. https://doi.org/ S0092-8674(05)00198-4.

27. Guertin DA, Stevens DM, Thoreen CC, Burds AA, Kalaany NY, Moffat J, Brown M, Fitzgerald KJ, Sabatini DM. Ablation in mice of the mTORC components raptor, rictor, or mLST8 reveals that $\mathrm{mTORC} 2$ is required for signaling to Akt-FOXO and PKCalpha, but not S6K1. Dev Cell. 2006; 11:859-71. https://doi.org/S1534-5807(06)00459-X.

28. Benvenuto G, Li S, Brown SJ, Braverman R, Vass WC, Cheadle JP, Halley DJ, Sampson JR, Wienecke R, DeClue JE. The tuberous sclerosis-1 (TSC1) gene product hamartin suppresses cell growth and augments the expression of the TSC2 product tuberin by inhibiting its ubiquitination. Oncogene. 2000; 19:6306-16. https://doi.org/10.1038/ sj.onc.1204009.

29. Liu Q, Thoreen C, Wang J, Sabatini D, Gray NS. mTOR Mediated Anti-Cancer Drug Discovery. Drug Discov Today Ther Strateg. 2009; 6:47-55. https://doi.org/10.1016/j. ddstr.2009.12.001.

30. Guertin DA, Sabatini DM. Defining the role of mTOR in cancer. Cancer Cell. 2007; 12:9-22. https://doi.org/ S1535-6108(07)00151-1.

31. Awada A, Cardoso F, Fontaine C, Dirix L, De Greve J, Sotiriou C, Steinseifer J, Wouters C, Tanaka C, Zoellner U, Tang P, Piccart M. The oral mTOR inhibitor RAD001 (everolimus) in combination with letrozole in patients with advanced breast cancer: results of a phase I study with pharmacokinetics. Eur J Cancer. 2008; 44:84-91. https:// doi.org/10.1016/j.ejca.2007.10.003.

32. Amato RJ, Jac J, Giessinger S, Saxena S, Willis JP. A phase 2 study with a daily regimen of the oral mTOR inhibitor RAD001 (everolimus) in patients with metastatic clear cell renal cell cancer. Cancer. 2009; 115:2438-46. https://doi. org/10.1002/cncr.24280.

33. Sarbassov DD, Ali SM, Sengupta S, Sheen JH, Hsu PP, Bagley AF, Markhard AL, Sabatini DM. Prolonged rapamycin treatment inhibits mTORC2 assembly and Akt/PKB. Mol Cell. 2006; 22:159-68. https://doi.org/ S1097-2765(06)00218-8.

34. Sarbassov DD, Ali SM, Kim DH, Guertin DA, Latek RR, Erdjument-Bromage H, Tempst P, Sabatini DM. Rictor, a novel binding partner of mTOR, defines a 
rapamycin-insensitive and raptor-independent pathway that regulates the cytoskeleton. Curr Biol. 2004; 14:1296-302. https://doi.org/10.1016/j.cub.2004.06.054.

35. Ali SM, Sabatini DM. Structure of S6 kinase 1 determines whether raptor-mTOR or rictor-mTOR phosphorylates its hydrophobic motif site. J Biol Chem. 2005; 280:19445-8. https://doi.org/C500125200.

36. Hardie DG. AMPK and Raptor: matching cell growth to energy supply. Mol Cell. 2008; 30:263-5. https://doi. org/10.1016/j.molcel.2008.04.012.

37. Li ST, Chen NN, Qiao YB, Zhu WL, Ruan JW, Zhou XZ. SC79 rescues osteoblasts from dexamethasone though activating Akt-Nrf2 signaling. Biochem Biophys Res Commun. 2016; 479:54-60. https://doi.org/10.1016/j. bbrc.2016.09.027.

38. Zhang H, Liu YY, Jiang Q, Li KR, Zhao YX, Cao C, Yao J. Salvianolic acid A protects RPE cells against oxidative stress through activation of $\mathrm{Nrf} / \mathrm{HO}-1$ signaling. Free Radic Biol Med. 2014; 69:219-28. https://doi.org/10.1016/j. freeradbiomed.2014.01.025.

39. Li KR, Yang SQ, Gong YQ, Yang H, Li XM, Zhao YX, Yao J, Jiang Q, Cao C. 3H-1,2-dithiole-3-thione protects retinal pigment epithelium cells against Ultra-violet radiation via activation of Akt-mTORC1-dependent Nrf2-HO-1 signaling. Sci Rep. 2016; 6:25525. https://doi.org/10.1038/ srep25525.

40. Suzuki T, Yamamoto M. Molecular basis of the Keap1-Nrf2 system. Free Radic Biol Med. 2015; 88:93-100. https://doi. org/10.1016/j.freeradbiomed.2015.06.006.

41. Zhang Q, Pi J, Woods CG, Andersen ME. A systems biology perspective on Nrf2-mediated antioxidant response. Toxicol Appl Pharmacol. 2010; 244:84-97. https://doi. org/10.1016/j.taap.2009.08.018.

42. Almeida M, Han L, Ambrogini E, Weinstein RS, Manolagas SC. Glucocorticoids and tumor necrosis factor alpha increase oxidative stress and suppress Wnt protein signaling in osteoblasts. J Biol Chem. 2011; 286:44326-35. https:// doi.org/10.1074/jbc.M111.283481.
43. Sato AY, Tu X, McAndrews KA, Plotkin LI, Bellido T. Prevention of glucocorticoid induced-apoptosis of osteoblasts and osteocytes by protecting against endoplasmic reticulum (ER) stress in vitro and in vivo in female mice. Bone. 2015; 73C: 60-8. https://doi. org/10.1016/j.bone.2014.12.012.

44. Plotkin LI, Manolagas SC, Bellido T. Glucocorticoids induce osteocyte apoptosis by blocking focal adhesion kinase-mediated survival. Evidence for inside-out signaling leading to anoikis. J Biol Chem. 2007; 282:24120-30. https://doi.org/10.1074/jbc.M611435200.

45. Nguyen T, Nioi P, Pickett CB. The Nrf2-antioxidant response element signaling pathway and its activation by oxidative stress. J Biol Chem. 2009; 284:13291-5. https:// doi.org/10.1074/jbc.R900010200.

46. Khatri S, Yepiskoposyan H, Gallo CA, Tandon P, Plas DR. FOXO3a regulates glycolysis via transcriptional control of tumor suppressor TSC1. J Biol Chem. 2010; 285:15960-5. https://doi.org/10.1074/jbc.M110.121871.

47. Li L, Zhu K, Liu Y, Wu X, Wu J, Zhao Y, Zhao J. Targeting thioredoxin-1 with siRNA exacerbates oxidative stress injury after cerebral ischemia/reperfusion in rats. Neuroscience. 2015; 284:815-23. https://doi.org/10.1016/j. neuroscience.2014.10.066.

48. Gong YQ, Huang W, Li KR, Liu YY, Cao GF, Cao C, Jiang Q. SC79 protects retinal pigment epithelium cells from UV radiation via activating Akt-Nrf2 signaling. Oncotarget. 2016; 7:60123-32. https://doi.org/10.18632/ oncotarget.11164.

49. Busch S, Auth E, Scholl F, Huenecke S, Koehl U, Suess B, Steinhilber D. 5-lipoxygenase is a direct target of miR19a-3p and miR-125b-5p. J Immunol. 2015; 194:1646-53. https://doi.org/10.4049/jimmunol.1402163.

50. Romaker D, Kumar V, Cerqueira DM, Cox RM, Wessely O. MicroRNAs are critical regulators of tuberous sclerosis complex and mTORC1 activity in the size control of the Xenopus kidney. Proc Natl Acad Sci USA. 2014; 111:6335-40. https://doi.org/10.1073/pnas.1320577111. 